\title{
Nonrelativistic effective field theory for meson-loop effects in heavy quarkonia
}

\author{
Feng-Kun Guo*† \\ Helmholtz-Institut für Strahlen- und Kernphysik and Bethe Center for Theoretical Physics, \\ Universität Bonn, D-53115 Bonn, Germany \\ E-mail: fkguo@hiskp.uni-bonn.de
}

\begin{abstract}
It was proposed that some of the transitions between two heavy quarkonia are dominated by coupled-channel effects, which are due to intermediate heavy meson and anti-meson loops. Such effects may be studied employing a nonrelativistic effective field theory. Here we review the power counting scheme and a few applications.
\end{abstract}

Xth Quark Confinement and the Hadron Spectrum,

October 8-12, 2012

TUM Campus Garching, Munich, Germany

\footnotetext{
* Speaker.

${ }^{\dagger}$ The work reported here is partially by the DFG and the NSFC through funds provided to the Sino-German CRC 110 "Symmetries and the Emergence of Structure in QCD" and by the NSFC (Grant No. 11165005).
} 


\section{Introduction}

Being bound states of a heavy quark and an anti-heavy quark, heavy quarkonium provides a unique probe for understanding QCD. Being much heavier than the nonperturbative scale $\Lambda_{\mathrm{QCD}}$, the heavy quarks inside a heavy quarkonium are nonrelativistic, and the heavy quarkonium spectroscopy below the open-flavour threshold can be well described in the framework of potential models, see, for instance [1]. However, mainly due to the running of the $B$-factories, quite a few new structures in the charmonium mass region were observed in recent years, the most famous one of which is the $X(3872)$ [2]. Most of them are above the $D \bar{D}$ threshold, and do not fit the expectations of the potential models. For a comprehensive review of their properties and models describing them, we refer to Ref. [3]. Understanding these new structures require the inclusion of the open-charm channels. This strategy was taken in, e.g., Ref. [4]. On the other hand, there are also evidences for the importance of the coupled-channel effects in transitions of heavy quarkonia, see, e.g. Ref. [5].

It is thus of high interest to have a theoretical framework to study the coupled-channel effects with controlled uncertainties. Such an approach, a nonrelativistic effective field theory (NREFT), was proposed in Ref. [6], and studied in detail later [7]. The same approach has been applied to some heavy quarkonium transitions, see Refs. $[8,9,10,11,12,13]$. Here, we will review the power counting scheme of NREFT, and a few applications of it. The power counting scheme of one-loop diagrams will be reviewed in Section 2. In Section 3, two groups of heavy quarkonium transitions will be discussed in NREFT, which include the transitions with the emission of one light pseudoscalar meson, and the hindered M1 radiative transitions between $P$-wave charmonia. We will show that in many of the processes, the coupled-channel effects play an important role, and even provide the dominant decay mechanism. A brief summary is given in Section 4.

\section{Power counting in NREFT}

When allowed by symmetries, coupled-channel effects always exist for a given process. Considering a radiative charmonium transition into another charmonium, it can of course occur directly by emitting a photon from one of the charm quarks, which is the case calculated in various quark models. It can also take place through coupling the initial and final charmonia to a pair of openflavor charmed and anti-charmed mesons, and the photon being emitted from one of the intermediate charmed mesons. This was considered, for instance, for the M1 transitions of the $S$-wave charmonia in an effective Lagrangian approach [14]. In that paper, the authors calculated the loops using form factors with a cut-off, and the results depend on the value of the cut-off. When one has well-separated scales, an effective field theory would be better since the uncertainty can be controlled.

For most heavy quarkonium transitions, we notice that $M_{Q \bar{Q}}-2 M_{Q \bar{q}} \ll M_{Q \bar{q}}$, where $M_{Q \bar{Q}}$ and $M_{Q \bar{q}}$ are the masses of the heavy quarkonium and an open-flavor heavy meson, respectively. As a result, the intermediate heavy mesons are nonrelativistic with a small velocity

$$
v \sim \sqrt{\left|M_{Q \bar{Q}}-2 m_{Q \bar{q}}\right| / m_{Q \bar{q}}} \ll 1 .
$$

Thus, one may construct a power counting in terms of $v$. To show how the power counting is organized, it is instructive to focus on an explicit example. Let us look at a three-point loop diagram, 


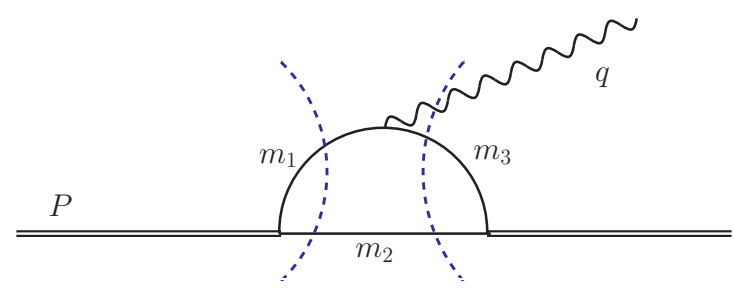

Figure 1: Three-point hadronic loop diagram. Double, solid and wiggly lines denote the heavy quarkonia, heavy mesons, and photon / light meson, respectively. The dashed curves represent the unitary cuts.

see Fig. 1, which is typical for heavy quarkonium transitions with the emission of a light meson or a photon. The scalar loop integral is defined as

$$
\begin{aligned}
I(q) & =i \int \frac{d^{4} l}{(2 \pi)^{4}} \frac{1}{\left(l^{2}-m_{1}^{2}+i \varepsilon\right)\left[(P-l)^{2}-m_{2}^{2}+i \varepsilon\right]\left[(l-q)^{2}-m_{3}^{2}+i \varepsilon\right]} \\
& \simeq \frac{i}{8 m_{1} m_{2} m_{3}} \int \frac{d^{4} l}{(2 \pi)^{4}} \frac{1}{\left[l^{0}-T_{1}(|\vec{l}|)\right]\left[P^{0}-l^{0}-T_{2}(|\vec{l}|)\right]\left[l^{0}-q^{0}-T_{3}(|\vec{l}-\vec{q}|)\right]},
\end{aligned}
$$

where $T_{i}(p)=p^{2} / 2 m_{i}=m_{i} v^{2} / 2$ denotes the kinetic energy for a heavy meson with mass $m_{i}$. In the second line, we have made a nonrelativistic expansion, and kept only the leading term. A shift in $l^{0}$ has been done so that the energy is of order $\vec{l}^{2} / m_{i}$. Thus, one may count the nonrelativistic momentum and energy in the powers of velocity as $v$ and $v^{2}$, respectively. Accordingly, the measure of the loop integral contributes $v^{5}$ to the velocity counting, and any of the propagators counts as $v^{-2}$. Therefore, we have $I(q) \sim v^{5} /\left(v^{2}\right)^{3}=v^{-1}$. This means that when the scalar three-point loop contributes, it is more important for smaller $v$.

To understand better this enhancement, we work out the loop function. Using dimensional regularization, the loop is convergent in four dimensions, and the analytic expression reads as

$$
I(q)=\mathscr{N} \frac{1}{\sqrt{a}}\left[\arctan \left(\frac{c^{\prime}-c}{2 \sqrt{a(c-i \varepsilon)}}\right)+\arctan \left(\frac{2 a+c-c^{\prime}}{2 \sqrt{a\left(c^{\prime}-a-i \varepsilon\right)}}\right)\right],
$$

where $\mathscr{N}=\mu_{12} \mu_{23} /\left(16 \pi m_{1} m_{2} m_{3}\right)$ with $\mu_{i j}=m_{i} m_{j} /\left(m_{i}+m_{j}\right)$ the reduced mass. Furthermore, we have defined $a=\left(\mu_{23} / m_{3}\right)^{2} \vec{q}^{2}, c=2 \mu_{12} b_{12}$, and $c^{\prime}=2 \mu_{23} b_{23}+\left(\mu_{23} / m_{3}\right) \vec{q}^{2}$, where $b_{12}=$ $m_{1}+m_{2}-M$ and $b_{23}=m_{2}+m_{3}+q^{0}-M$ with $M$ the mass of the initial particle. The two arctan functions correspond to the two unitary cuts depicted in Fig. 1, and the loop function is the sum of contributions of these two cuts. Accordingly, one may define two velocities of the intermediate mesons: $v_{1}=\sqrt{c / m}$ and $v_{2}=\sqrt{\left(c^{\prime}-a\right) / m}$ with $m \simeq m_{i}$. Eq. (2.3) suggests that the velocity $v$ in NREFT power counting should be understood as the average of these two velocities. It becomes more clear if we expand the expression in the following way

$$
I(q)=\mathscr{N} \frac{2}{\sqrt{c^{\prime}-a}+\sqrt{c}}\left[1+\mathscr{O}\left(\frac{a}{\min \left(c^{\prime},\left(c^{\prime}-c\right) / 2\right)}\right)\right] .
$$

The expansion is valid for all the transitions studied here. 


\section{Applications}

In this section, we will review the applications of NREFT to two kinds of heavy quarkonium transitions: hadronic transitions with the emission of a light pseudoscalar meson and the hindered M1 radiative transitions between charmonia. Interesting physics such as the extraction of the value of $m_{u} / m_{d}$ and chiral extrapolation in heavy quarkonium transitions will also be discussed.

\subsection{Hadronic transitions between heavy quarkonia}

Here, the transitions to be discussed are hadronic transitions emitting a pion or an eta. The former breaks isospin symmetry, while the latter breaks SU(3) flavor symmetry. These transitions can certainly receive contributions from hadronic loops, and the power counting of the transitions should be analyzed case by case (for more details, see Ref. [7]).

In the hadronic loop mechanism, the flavor symmetry breaking is due to the mass difference between mesons containing different light quarks. For instance, both the charged and neutral charmed meson loops contribute to charmonium transitions, and their difference due to nonvanishing $M_{D^{+}}-M_{D^{0}}$ induces isospin breaking. Similarly, $M_{D_{s}}-M_{D}$ results in SU(3) breaking. Denoting the meson mass difference by $\Delta$, it gives a contribution of $\Delta / v^{2}$ to the amplitude. Here $1 / v^{2}$ is introduced for balance because we have split out $\Delta$, a small energy scale. This can be shown by expanding the propagator of the charged $D$-meson [12],

$$
\frac{1}{l^{0}-\vec{l}^{2} /\left(2 m_{D}\right)+\Delta}=\frac{1}{l^{0}-\vec{l}^{2} /\left(2 m_{D}\right)}-\frac{\Delta}{\left[l^{0}-\vec{l}^{2} /\left(2 m_{D}\right)\right]^{2}}+\ldots
$$

and the leading term will be cancelled with the neutral meson loop.

To get the complete power counting of a given loop diagram, in addition to counting the scalar loop in the previous section and the factor $\Delta v^{-2}$, one still needs to consider the involved vertices. Because of chiral symmetry, the pionic vertex contributes a factor of external momentum at leading order, denoted by $\vec{q}$. The coupling of a heavy quarkonium to a heavy meson and anti-meson pair can be in either an $S$ wave or a $P$ wave, depending on the quantum numbers of the heavy quarkonium - the $S$-wave ( $P$-wave) heavy quarkonium couples to heavy mesons in a(n) $P(S)$ wave (only the ground state heavy mesons are considered here). Hence, the transitions can be organized in three groups, and the power counting of each is given as follows.

1) Both the initial and final heavy quarkonia are $S$-wave states. In this case, both the $P$-wave vertices contribute an internal momentum. In total, they give a contribution of $v^{2}$. In this case, the amplitude of the three-point diagram scales as $\Delta|\vec{q}| / v .{ }^{1}$ Comparing with the treelevel amplitude, which scales as $\delta|\vec{q}|$ with $\delta \sim \Delta$ the quark mass difference, the triangle loops thus give an enhancement especially for small values of $v$.

2) Both heavy quarkonia are $P$-wave states. In this case, both the vertices are in an $S$ wave. The amplitude of such a diagram counts as $\Delta|\vec{q}| / v^{3}$, and provides a stronger enhancement than the previous case.

\footnotetext{
${ }^{1}$ Notice that in this power counting, we have neglected the coupling constants which are complicated functions of the heavy quark mass, heavy quark velocity $v_{Q}$ and $\Lambda_{\mathrm{Q} C D}$. We are not aware of any model-independent analysis of the dependence of these quantities on various energy scales. For a discussion using model expressions of the coupling constants, we refer to Ref. [7].
} 


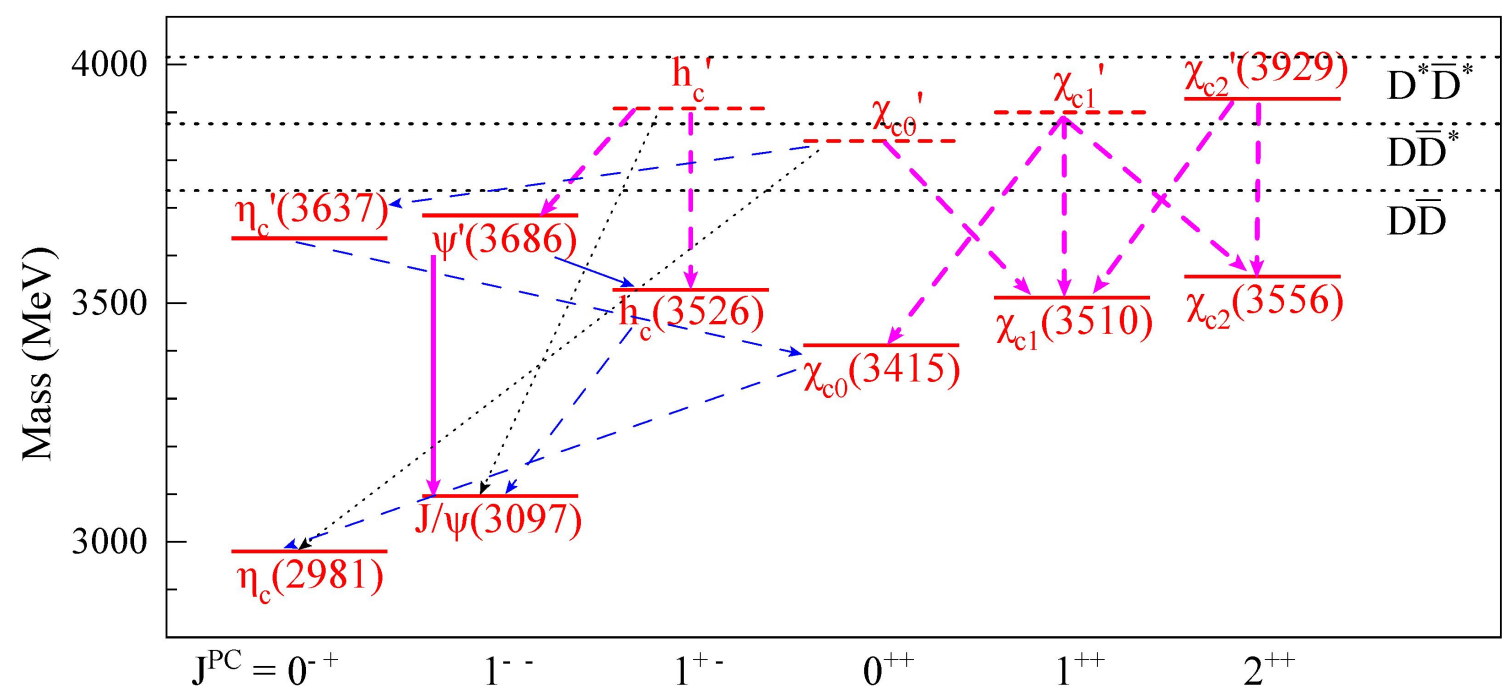

Figure 2: Possible $S$ and $P$-wave charmonium transitions with the emission of one pion or $\eta$ meson. The dashed lines represent the resonances, for which potential model predictions of the masses [16] are used, and decay modes which have not been observed so far. The dotted horizontal lines denote the $D \bar{D}, D \bar{D}^{*}$ and $D^{*} \bar{D}^{*}$ thresholds. The measured decays are represented by the solid lines, and the dotted lines are for the decays beyond the range of the applicability of NREFT. Among the lines with arrows, the thicker ones indicate transitions with enhanced meson loops.

3) One of the heavy quarkonia is a $P$-wave state, and the other an $S$-wave state. Only the $P$ wave vertex is linear in momentum, and the other contributes a constant factor. However, this momentum should be counted as $|\vec{q}|$ instead of $v$ because it has to contract with the external momentum $\vec{q}$ into a Galilean invariant quantity. The loop amplitude counts as $\Delta \vec{q}^{2} /\left(M_{D}^{2} v^{3}\right)$, where $1 / M_{D}^{2}$ is introduced to match dimensions since the tree-level amplitude scales as $\delta$.

All possible transitions involving the ground state and first radially excited charmonia are summarized in Fig. 2.

An immediate consequence is that the $\psi^{\prime} \rightarrow J / \psi \eta / \pi^{0}$ transitions receive important contribution from the coupled-channel effects. Therefore, the long-standing suggestion that these transitions can be used to extract the ratio of the up and down quark masses [15] need to be reexamined. In fact, if we assumes that the triangle loop diagrams saturate the transitions, the resulting prediction of $\mathscr{B}\left(\psi^{\prime} \rightarrow J / \psi \pi^{0}\right) / \mathscr{B}\left(\psi^{\prime} \rightarrow J / \psi \eta\right)$ is consistent with the experimental data. These transitions were revisited considering both the loop and tree diagrams in Ref. [12]. From the above analysis, one sees that not all of the transitions have enhanced coupled-channel effects. For instance, the $\Upsilon(4 S) \rightarrow h_{b} \eta / \pi^{0}$ should be dominated by the tree-level contribution, and can be used for the quark mass ratio extraction [9].

\subsection{Hindered M1 transitions between $P$-wave heavy quarkonia}

Further insight into the coupled-channel effects in heavy quarkonium transitions can be gained from the radiative transitions. Especially, we find that the hindered M1 transitions between two $P$ wave charmonia, such as $\chi_{c J}(2 P) \rightarrow \gamma h_{c}(1 P)$ and $h_{c}(2 P) \rightarrow \gamma \chi_{c J}(1 P)$, should be dominated by the 

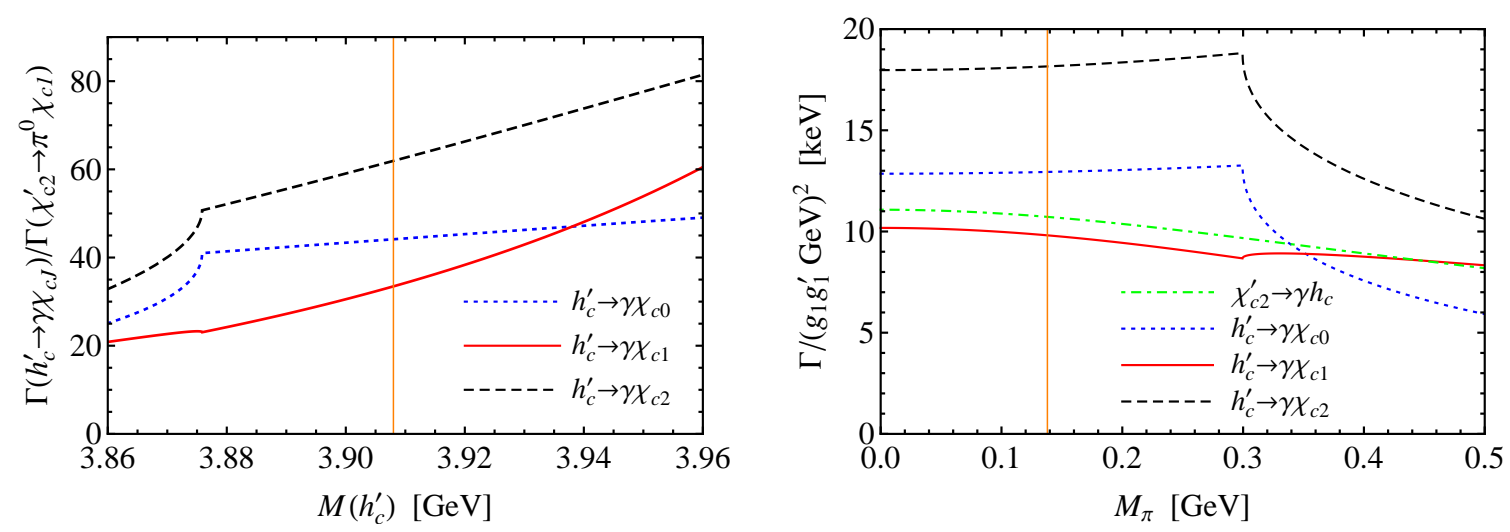

Figure 3: Left: Parameter-free ratios $\Gamma\left(h_{c}^{\prime} \rightarrow \gamma \chi_{c J}\right) / \Gamma\left(\chi_{c 2}^{\prime} \rightarrow \pi^{0} \chi_{c 1}\right)$, where the vertical line denotes the model value of the $h_{c}^{\prime}$ mass $3.908 \mathrm{GeV}$ calculated in Ref. [16]. Right: Pion mass dependence of the widths of various hindered M1 transitions, where the vertical line denotes the physical pion mass.

coupled-channel effects. In a quark model, the amplitude for such a transition is proportional to the overlap of the wave functions of the initial and final charmonia. It is nonzero just because of small relativistic corrections. In potential nonrelativistic QCD, the leading order amplitude would be of order $E_{\gamma} v_{c} / m_{c}$ [17], where $v_{c}$ is the charm quark velocity. The amplitude vanishes if the charm quark is infinitely heavy because a transition between a spin-triplet and singlet states flips the polarization of a heavy quark, and such a spin-flip is suppressed in the heavy quark limit. If the transitions happen through the charmed meson loops, the leading contribution would be of order $I(q) E_{\gamma} / m_{c} \sim E_{\gamma} /\left(m_{c} v\right)$. Therefore, the hindered M1 transitions between two $P$-wave charmonia are dominated by the coupled-channel effects. Because the loops are convergent, it is straightforward to calculate the widths. The results depend on a product of two unknown parameters for the coupling of the $1 P$ and $2 P$ charmonia to the charmed mesons. If we take model values for them, the decay width of the $\chi_{c 2}(2 P) \rightarrow \gamma h_{c}(1 P)$ is of $\mathscr{O}(100 \mathrm{keV})$, which is two orders of magnitude larger than the quark model prediction, $1.3 \mathrm{keV}$ [18].

As discussed in the previous section, the transitions between two $P$-wave charmonia emitting a pion should also be dominated by the charmed meson loops. Their decay amplitudes are proportional to the same product of unknown coupling constants. Therefore, ratios of the decay widths of these two different types of transitions can be predicted parameter free. The results are shown in the left panel of Fig. 3. The cusps on the curves are due to the opening of the $D \bar{D}^{*}$ threshold.

There have been lattice calculations of the radiative transitions of heavy quarkonia. In order to reduce computational cost, unphysical light quark masses are used in these calculations so that the pion mass is heavier than its physical value. For instance, the calculations of the M1 transitions of the $S$-wave charmonia in Ref. [19] use a pion mass of $485 \mathrm{MeV}$. An intriguing consequence of our analysis is that the decay widths could change a lot when varying the pion mass if the transitions are dominated by the coupled-channel effects. It is because the quark mass dependence can arise from the light quarks in the intermediate charmed mesons. Therefore, proper chiral extrapolation is necessary to get the physical results. For illustration, the right panel of Fig. 3 shows the predicted pion mass dependence of the decay widths of several hindered M1 transitions, where we have used $3.908 \mathrm{GeV}$ predicted in Ref. [16] for the $h_{c}(2 P)$ mass. One sees that the result at a pion mass of 
$485 \mathrm{MeV}$ could deviate from the physical value by a factor of 2 . The cusps on the curves are due to crossing the $D \bar{D}^{*}$ threshold.

Can similar predictions be made for the bottomonium transitions? To answer this question, one has to scrutinize higher order corrections, and check whether they are suppressed as in the charmonium counterparts. As shown in Ref. [11], the next-to-leading corrections to the one-loop triangle diagrams come from two loops with the exchange of one pion. They are suppressed comparing to the leading contribution by a factor of $v\left(g M_{D} / \Lambda_{\chi}\right)^{2} \sim 0.4$, where $g \simeq 0.6$ is the axial coupling constant in the charmed sector, and $\Lambda_{\chi} \simeq 1 \mathrm{GeV}$ is the chiral symmetry breaking scale. Replacing $M_{D}$ by $M_{B}$, one would have $v\left(g M_{B} / \Lambda_{\chi}\right)^{2}>1$. Thus, the diagrams with more loops are not suppressed relative to the one-loop diagrams, and quantitative predictions cannot be made. However, one may still conclude that the coupled-channel effects dominate the hindered M1 transitions between two $P$-wave bottomonia.

\section{Summary}

We have shown that for heavy quarkonium transitions with a small phase space, the coupledchannel effects, due to the creation and annihilation of a pair of intermediate heavy meson and anti-meson, can be investigated using a nonrelativistic effective field theory. The power counting scheme in terms of nonrelativistic velocities is described at the one-loop level. For discussions of two and more loops, we refer to Refs. [10, 11, 20]. NREFT was then applied to both hadronic and radiative transitions of heavy quarkonia. We show that the coupled-channel effects play a very important role in some charmonium transitions emitting a pion. In particular, the $\psi^{\prime} \rightarrow J / \psi \eta / \pi^{0}$ cannot be used to extract the light quark mass ratio. Instead, the $\Upsilon(4 S) \rightarrow h_{b} \eta / \pi^{0}$ are better for this purpose in the sense that the hadronic loops will not introduce a sizable correction. We also suggest that the hindered M1 transitions between two $P$-wave charmonia are dominated by the coupled-channel effects. As a result of the light quarks inside the intermediate charmed mesons, the decay widths of these transitions have a strong and even nonanalytic dependence on the pion mass. Quantitative predictions were made, and they should be confronted with further experimental investigations and lattice calculations.

\section{References}

[1] S. Godfrey and N. Isgur, Mesons in a relativized quark model with chromodynamics, Phys. Rev. D 32 (1985) 189.

[2] Belle Collaboration, S. Choi et al., Observation of a narrow charmonium - like state in exclusive $B^{ \pm} \rightarrow K^{ \pm} \pi^{+} \pi^{-} J / \psi$ decays, Phys. Rev. Lett. 91 (2003) 262001, [hep-ex/ 0309032 ].

[3] N. Brambilla, S. Eidelman, B. Heltsley, R. Vogt, G. Bodwin, et al., Heavy quarkonium: progress, puzzles, and opportunities, Eur. Phys. J. C 71 (2011) 1534, [arXiv: 1010.5827 ].

[4] E. J. Eichten, K. Lane, and C. Quigg, New states above charm threshold, Phys. Rev. D 73 (2006) 014014, [hep-ph/0511179].

[5] Y.-J. Zhang, G. Li, and Q. Zhao, Manifestation of intermediate meson loop effects in charmonium decays, Chin. Phys. C 34 (2010) 1181. 
[6] F.-K. Guo, C. Hanhart, and U.-G. Meißner, On the extraction of the light quark mass ratio from the decays $\psi^{\prime} \rightarrow J / \psi \pi^{0}(\eta)$, Phys. Rev. Lett. 103 (2009) 082003, [arXiv: 0907.0521$]$.

[7] F.-K. Guo, C. Hanhart, G. Li, U.-G. Meißner, and Q. Zhao, Effect of charmed meson loops on charmonium transitions, Phys. Rev. D 83 (2011) 034013, [arXiv: 1008 . 3632].

[8] F.-K. Guo, C. Hanhart, G. Li, U.-G. Meißner, and Q. Zhao, Novel analysis of the decays $\psi^{\prime} \rightarrow h_{c} \pi^{0}$ and $\eta_{c}^{\prime} \rightarrow \chi_{c 0} \pi^{0}$, Phys. Rev. D 82 (2010) 034025, [arXiv: 1002.2712 ].

[9] F.-K. Guo, C. Hanhart, and U.-G. Meißner, Extracting the light quark mass ratio $m_{u} / m_{d}$ from bottomonia transitions, Phys. Rev. Lett. 105 (2010) 162001, [arXiv: 1007 . 4682].

[10] M. Cleven, F.-K. Guo, C. Hanhart, and U.-G. Meißner, Bound state nature of the exotic $Z_{b}$ states, Eur. Phys. J. A 47 (2011) 120, [arXiv:1107.0254].

[11] F.-K. Guo and U.-G. Meißner, Examining coupled-channel effects in radiative charmonium transitions, Phys. Rev. Lett. 108 (2012) 112002, [arXiv:1111.1151].

[12] T. Mehen and D.-L. Yang, On the role of charmed meson loops in charmonium decays, Phys. Rev. D 85 (2012) 014002, [arXiv:1111.3884].

[13] F.-K. Guo and U.-G. Meißner, Light quark mass dependence in heavy quarkonium physics, Phys. Rev. Lett. 109 (2012) 062001, [arXiv:1203.1116].

[14] G. Li and Q. Zhao, Hadronic loop contributions to $J / \psi$ and $\psi^{\prime}$ radiative decays into $\gamma \eta_{c}$ or $\gamma \eta_{c}^{\prime}$, Phys. Lett. B 670 (2008) 55-60, [arXiv:0709.4639].

[15] B. Ioffe, Masses of light quarks and interaction of low-energy $\eta$ mesons (in Russian), Yad. Fiz. 29 (1979) 1611.

[16] B.-Q. Li and K.-T. Chao, Higher charmonia and X,Y,Z states with screened potential, Phys. Rev. D 79 (2009) 094004, [arXiv:0903.5506].

[17] N. Brambilla, Y. Jia, and A. Vairo, Model-independent study of magnetic dipole transitions in quarkonium, Phys. Rev. D 73 (2006) 054005, [hep-ph/ 0512369 ].

[18] T. Barnes, S. Godfrey, and E. Swanson, Higher charmonia, Phys. Rev. D 72 (2005) 054026, [hep-ph/0505002].

[19] Y. Chen, D.-C. Du, B.-Z. Guo, N. Li, C. Liu, et al., Radiative transitions in charmonium from $N_{f}=2$ twisted mass lattice QCD, Phys. Rev. D 84 (2011) 034503, [arXiv: 1104.2655$].$

[20] M. Cleven, Q. Wang, F.-K. Guo, C. Hanhart, U.-G. Meißner, and Q. Zhao, in preparation. 